\title{
On the Iteration of a Rational Function: Computer Experiments with Newton's Method
}

\author{
James H. Curry ${ }^{1}$, Lucy Garnett ${ }^{2}$, and Dennis Sullivan ${ }^{3}$ \\ 1 Department of Mathematics, University of Colorado, Boulder, CO 80309, USA \\ 2 Lucy Garnett, Department of Statics and Computers, Baruch College, 17 Lexington Avenue, \\ New York, NY 10010, USA \\ 3 Graduated School and University Center, CUNY, New York, NY and \\ I.H.E.S., F-91440, Bures-sur-Yvette, France
}

\begin{abstract}
Using Newton's method to look for roots of a polynomial in the complex plane amounts to iterating a certain rational function. This article describes the behavior of Newton iteration for cubic polynomials. After a change of variables, these polynomials can be parametrized by a single complex parameter, and the Newton transformation has a single critical point other than its fixed points at the roots of the polynomial. We describe the behavior of the orbit of the free critical point as the parameter is varied. The Julia set, points where Newton's method fail to converge, is also pictured. These sets exhibit an unexpected stability of their gross structure while the changes in small scale structure are intricate and subtle.
\end{abstract}

\section{Introduction}

The study of iteration of rational mappings of a complex variable has a long history. Seminal work on this topic appeared in the early studies of Fatou and Julia at the turn of the $20^{\text {th }}$ century $[4,7]$. In the $60^{\text {'s }}$ the work of Brolin [1] and Guckenheimer [5], Jakobson [6] should be mentioned. More recently Sullivan [10] Mané et al. [9] have made contributions. The articles cited above have made significant theoretical contributions; in contrast there have been few experimental studies of the iterates of rational maps. The work of Mandelbrot [8] has stood alone.

Mandelbrot has considered iteration of the transformation

$$
f_{c}(z)=z^{2}+c
$$

where $c$ is a complex number, and produced striking pictures whose most obvious feature is the prevailing self-similarity. A major contribution toward the understanding of Mandelbrot's bifurcation diagram has been announced by Douady and Hubbard $[2,3]$. 
The quadratic transformation (1.1) is perhaps the simplest nontrivial rational map. We will study here another class of examples, rational maps contructed by applying Newton's method to a polynomial $f(z)$ :

$$
N f(z)=z-\frac{f(z)}{f^{\prime}(z)}
$$

We will refer to $N f(z)$ as the Newton Transformation associated with the function $f(z)$.

The transformation (1.2) has been and is much studied by numerical mathematicians whose primary concern is with choosing initial conditions so that the iteration sequence

$$
z_{n+1}=z_{n}-\frac{f\left(z_{n}\right)}{f^{\prime}\left(z_{n}\right)}
$$

converges to a solution of the equation $f(z)=0$. These studies have generally not attempted to catalogue all the possible types of behavior that the sequence of points $\left\{z_{n}\right\}$ may exhibit. On the other hand, the classical theory due to Fatou and Julia offers some general insight into the possible behaviors of the sequence $\left\{z_{n}\right\}$. The purpose of this article is to catalogue in a concrete way, using the computer, the asymptotic behavior of (1.3) when $f(z)$ is a cubic polynomial with simple roots.

In Sect. 2 the precise family of polynomials to be considered is presented along with other preliminary results. Section 3 discusses the morphology in parameter space of the Newton transformation as parameters are changed. In Sect. 4 the Julia sets from a random walk in parameter space are presented and the asymptotic behavior of the free critical point is described.

\section{Preliminaries}

We study

$$
N f_{A}(z)=z-f_{A}(z) / f_{A}^{\prime}(z)
$$

where $f_{A}(z)$ is the cubic polynomial

$$
f_{A}(z)=z^{3}+(A-1) z-A .
$$

The polynomials $f_{A}$ are exactly the monic cubics whose roots sum to zero and which have 1 as a root. Since any cubic can be transformed into an $f_{A}$ or into $z^{3}$ by an affine change of variable and multiplication by a constant, analyzing Newton's method for a general cubic reduces essentially to analyzing it for the $f_{A}$ 's.

In what follows the critical points of $N f_{A}(z)$ will play a crucial role. The critical points of a function are simply those points where its derivative vanishes. In particular, since the derivative of $(2.1)$ is given by

$$
\left(N f_{A}(z)\right)^{\prime}=\frac{f_{A}(z) f_{A}^{\prime \prime}(z)}{\left(f_{A}^{\prime}(z)\right)^{2}},
$$

the critical points are located at the zeros of $f_{A}(z)$ and $z=0$.

Much of the motivation for the material to be presented is the following theorem due to Fatou [4]: 
Theorem. If $R(z)$, a rational function, has an attracting periodic cycle, then the orbit of at least one critical point will converge to it.

Three of the critical points of $N f_{A}$ are fixed points so the fourth critical point located at $z=0$, is the only one available to converge to an attractive periodic cycle, should such a cycle exist. Hence, in what follows the orbit of the free critical point, at $z=0$, shall be our only concern.

The closure of the expanding periodic points, the sources, is called the Julia set of the transformation and will be denoted by $J\left(N f_{A}\right)$. We remark that the Julia set, which is closed and nowhere dense, together with the domain of an attractive periodic cycle (if there is one) constitute the set of initial points where Newton's method fails.

\section{Numerical Results: Morphology in Parameter Space}

Let us now describe the numerical experiments which were performed in the $A$-parameter plane. In order to determine whether a stable attracting periodic solution exists for (2.1) the theorem cited in the previous section indicates that it only necessary to follow the orbit of the free critical point. Typically, the initial condition $z=0$ was iterated as many as 200 times while simultaneously computing the norm of the difference of the current iterate and $z=1$. If the norm was less than $10^{-4}$, it was assumed that, for this $A$-parameter value, the orbit of the critical point converged to one. This procedure was used to test 160,000 parameter values which were elements of a square grid with 400 points in both horizontal and vertical directions.

In Fig. 3.1 the global behavior of orbit of the free critical point is pictured. The horizontal and vertical axes correspond to the real and imaginary parts of the complex parameter $A$. The dark area in this figure is the subset of 160,000 parameter values for which the orbit of the critical point tends to the root $z=1$.

Figure 3.2 is an enlargement of the region $[0.95,1.05] \times[-0.05,0.05]$. In this figure, which was generated by examining parameter values on a $400 \times 400$ grid, the self-similarity of regions in parameter space is evident. The "hole" in parameter space for $A=1$ is real since the orbit of the free critical point maps to infinity in one iteration for this $A$-value.

Figure 3.3 is an enlargement of the "island" at the top of Fig. 3.1. Once again the dark area in this figure corresponds to the set of parameter values for which the orbit of the free critical point tends to one. It is now necessary to distinguish between two kinds of white regions, those corresponding to parameter values where the orbit of the free critical point converges to a fixed point (i.e., a root of $f_{A}$ ) other than one, and those where the orbit of the free critical point is asymptotically periodic. The dark region in Fig. 3.4 represents the set of parameter values for which iterates of $z=0$ do not converge to a fixed point. In the central part of this figure the Newton transformation has a stable attractive period two cycle, e.g. $A=(0.31,1.62)$. In the large region to the left of the central part, e.g. $A=(0.275,1.65)$, Eq. $(2.1)$ has a stable period 4 cycle. For $A=(0.265,1.655)$ there is a stable period 8 cycle, as the real part of $A$ moves further to the left, but still remains within the dark region. Further period doublings have been observed, but the rate of accumulation of these bifurcations has not been examined. 


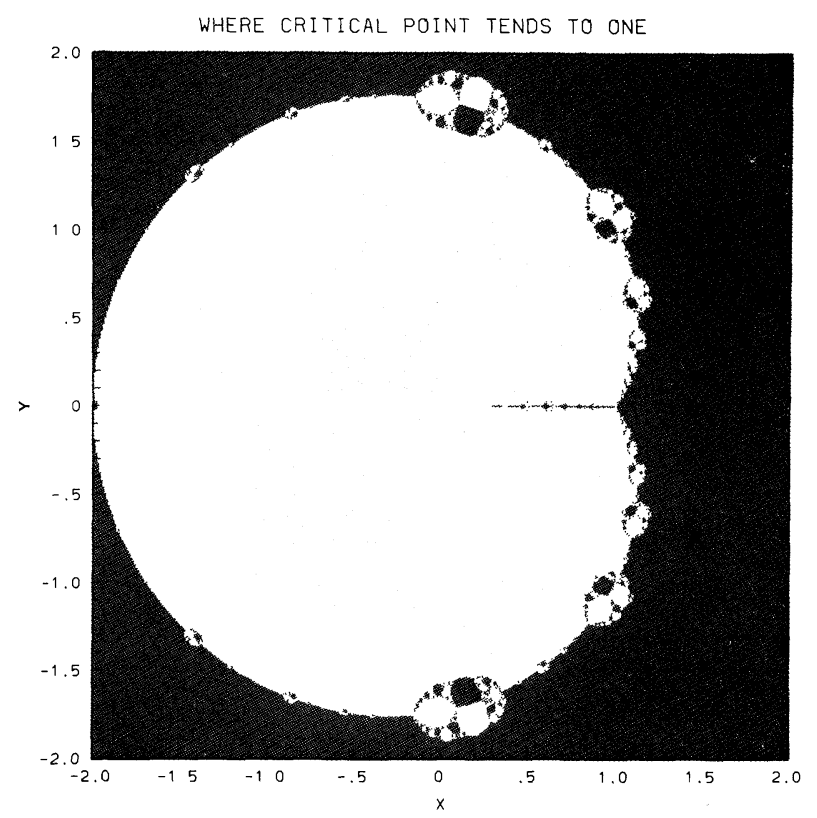

Fig. 3.1. Morphology in parameter space. Horizontal and vertical axes correspond to the real and imaginary parts of the complex parameter $A$

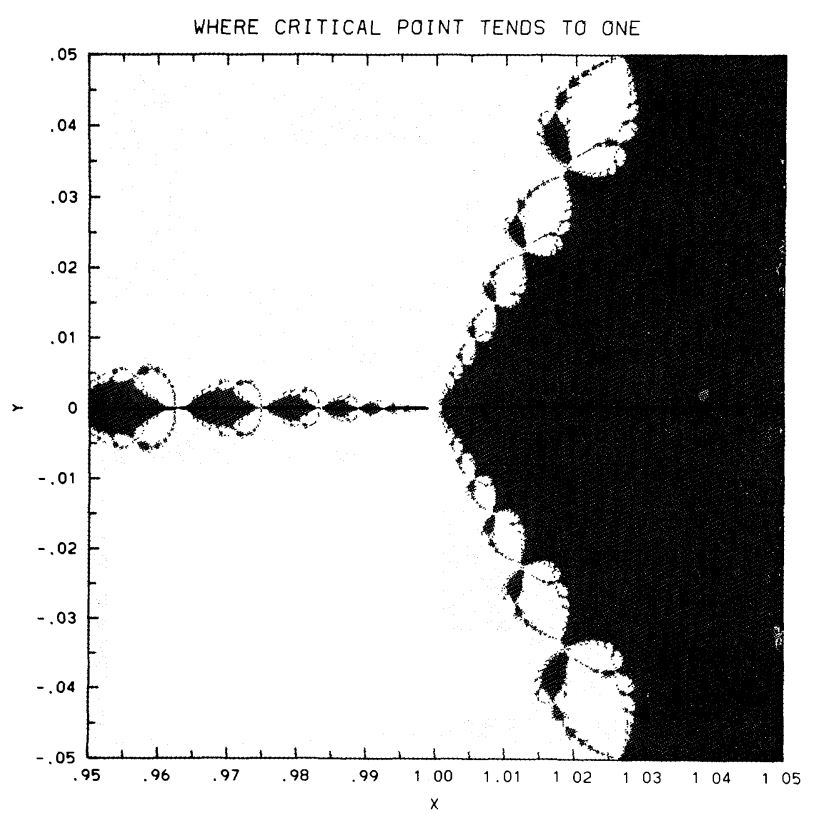

Fig. 3.2. Enlargement of the region $[0.95,1.05] \times[-0.05,0.05]$. Self-similarity in a neighborhood of $A=1$ is clearly visible 


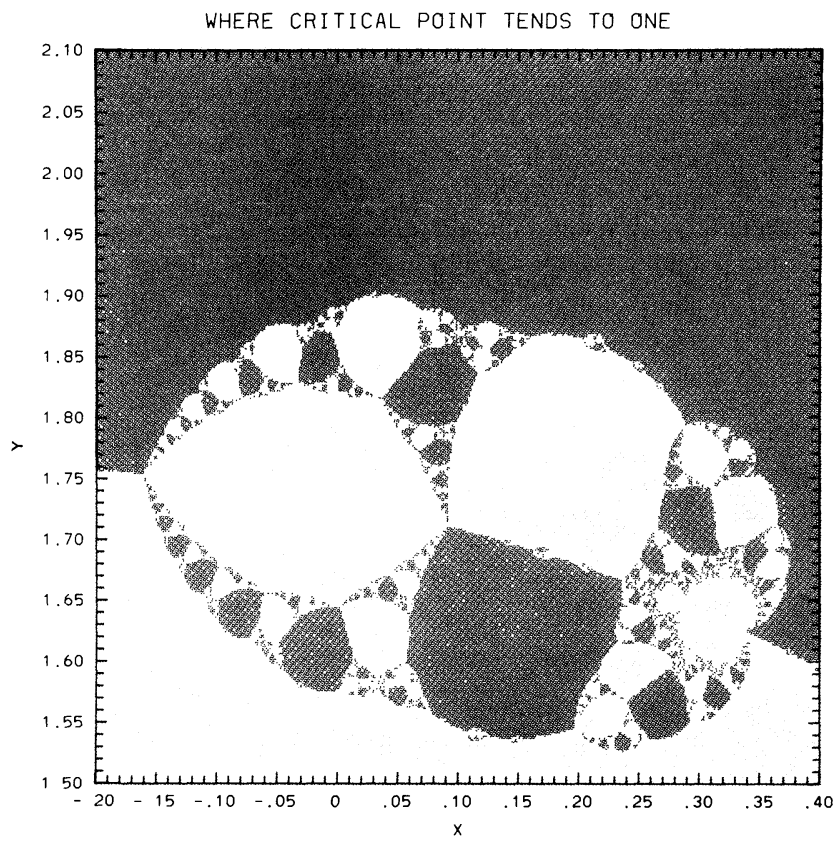

Fig. 3.3. Enlargement of the island at the top of Fig. 3.1

WHERE CRITICAL POINT IS PERIODIC

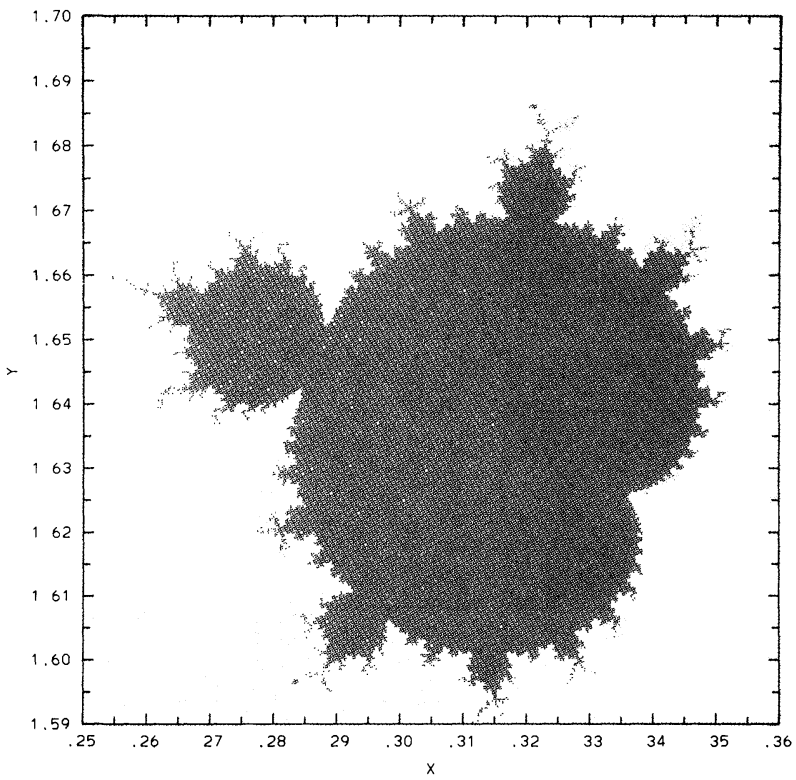

Fig. 3.4. One of the region where Newton's method exhibits periodic behavior 
If the central region is exited near the top of Fig. 3.4 period tripling has been observed $[A=(0.323,1.673)$ for period 6 and $(0.3225,1.67875)$ for period 12$]$ and quintupling bifurcations are also present in this system $[A=(0.3425,1.6625)$ and $A=(0.3025,1.6675)$, respectively].

To close this section we return to Fig. 3.1, where other islands are visible. Choosing successively the progressively smaller islands in a clockwise rotation from the topmost island, parameter regions for which the free critical point converges to periods $3,4,5,6$, and 7 have been observed. In each of these regions period doubling bifurcations are also present.

\section{A Walk in Parameter Space: Julia Sets}

As mentioned in Sect. 2, the Julia set, $J\left(N f_{A}\right)$, is the closure of the expanding periodic points. An approximation to $J\left(N f_{A}\right)$ can be computed by using the fact, due to Fatou and Julia, that for any $z$ in $J\left(N f_{A}\right)$ the inverse orbit $\bigcup_{n>0} N f_{A}^{-n}(z)$ is dense in $J\left(N f_{A}\right)$. Thus it is sufficient to locate a point $z \in J\left(N f_{A}\right)$ and compute its inverse orbit. The point at infinity is an obvious choice for $z$; it is a repelling fixed point, and its other two inverse images are the critical points of $f_{A}$. A tree structure is then generated which starts from a node (initially a critical point) and has three branches corresponding to the inverse images of that node. The tree is then allowed to grow to some maximum level, typically level 10, and the resulting points are plotted.

The complement of $J\left(N f_{A}\right)$ in the Riemann sphere, $\overline{\mathbb{C}}$, consist of countably many connected components, called the stable regions of $N f_{A}$ and are transformed among themselves by the action of the Newton map. Under iteration, each stable region is eventually cyclic and the cycles are classifiable into five types, Sullivan [10]. The two sorts of stable regions which are relevent for this section are the superattractive and attractive basins. An attractive basin consists of the components of the stable manifold of a periodic orbit and it is required that the modulus of the derivative at the periodic cycle is between zero and one (strict inequality). The superattractive basin is similarily defined but the modulus of the derivative at the cycle vanishes, e.g. at the fixed points of $N f_{A}$.

Table 1 is a tabulation of the asymptotic behavior of the orbit of the free critical point for the Julia sets which are presented in this section. As indicated by the parameter values in the table, attention is concentrated on values corresponding to points in the island pictured in Fig. 3.3. Clearly, it is expected that as

Table 1

\begin{tabular}{lcl}
\hline$A$-parameter values & Period & Modulus of derivative \\
\hline$(0.000,1.750)$ & 1 & 0.000 \\
$(0.210,1,760)$ & 1 & 0.000 \\
$(0.310,1.620)$ & 2 & 0.524 \\
$(0.275,1.650)$ & 4 & 0.259 \\
$(0.323,1.673)$ & 6 & 0.379 \\
$(0.305,1.667)$ & 440 & 0.820 \\
\hline
\end{tabular}




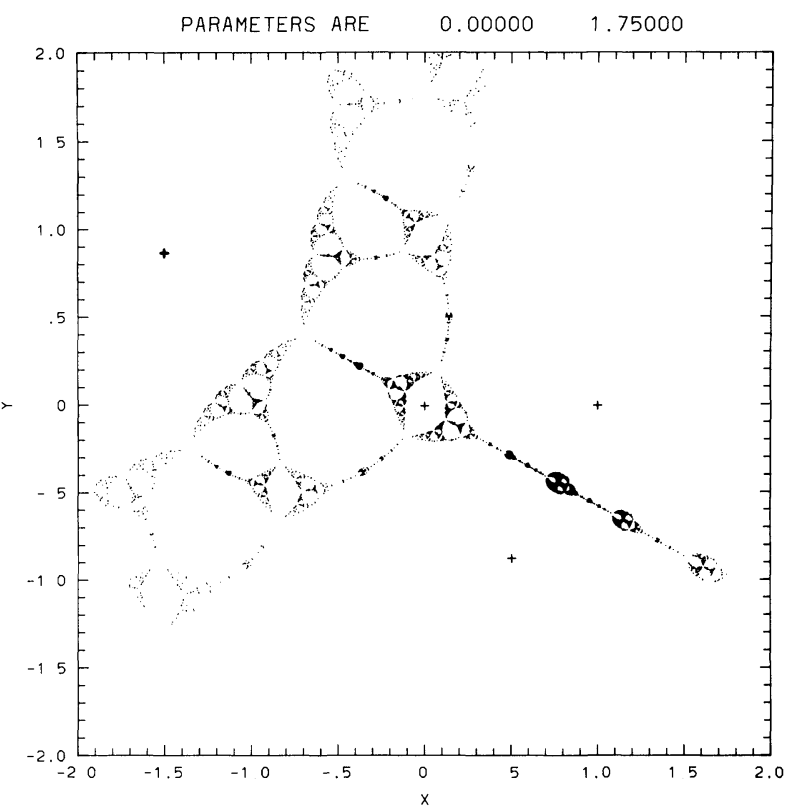

Fig. 4.1. A typical Julia set for parameter values in the region pictured in Fig. 3.2

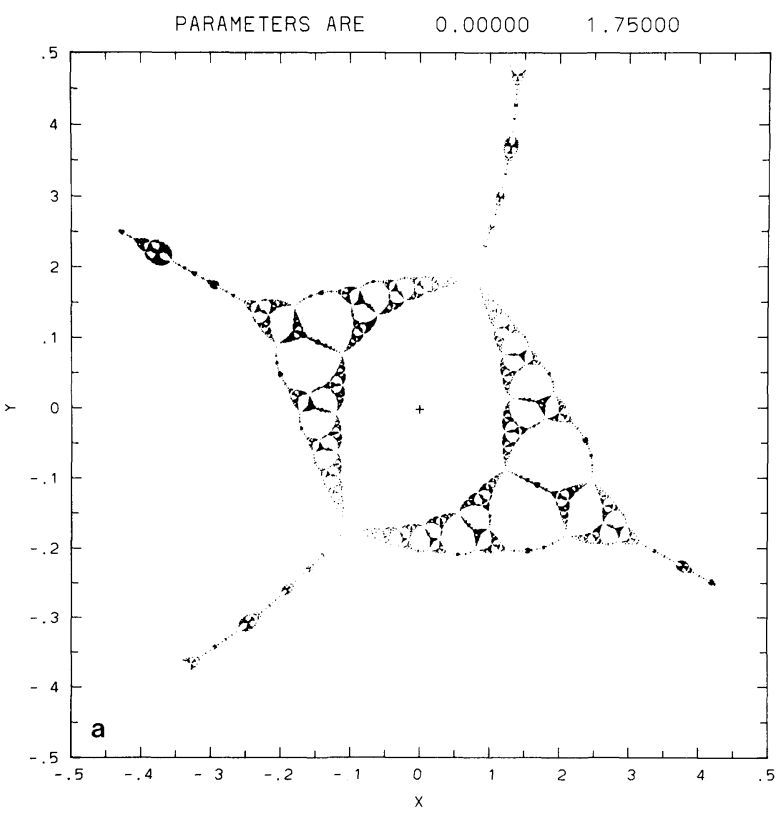

Fig. 4.2a-f. A random walk through parameter space 

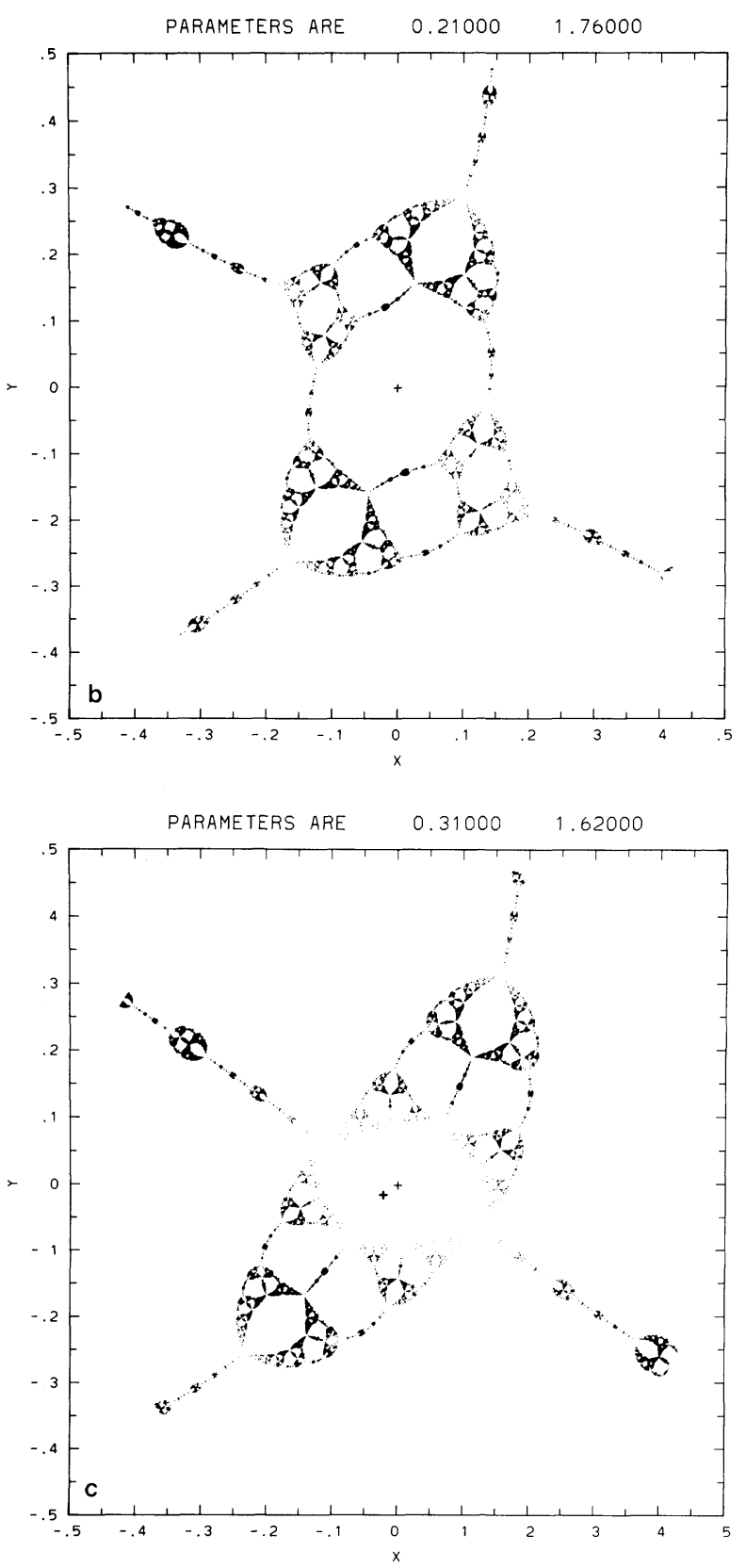

parameter values are varied the Julia set for $N f_{A}$ will undergo many structural changes, but the "gross" structure remains essentially unchanged once values are in the region pictured in Fig. 3.3. Figure 4.1 exhibits this typical structure.

In Fig. 4.2a-f attention is concentrated on the portion of $J\left(N f_{A}\right)$ which lies in the square $[-0.5,0.5] \times[-0.5,0.5]$. The cross(es) present in each of figures 

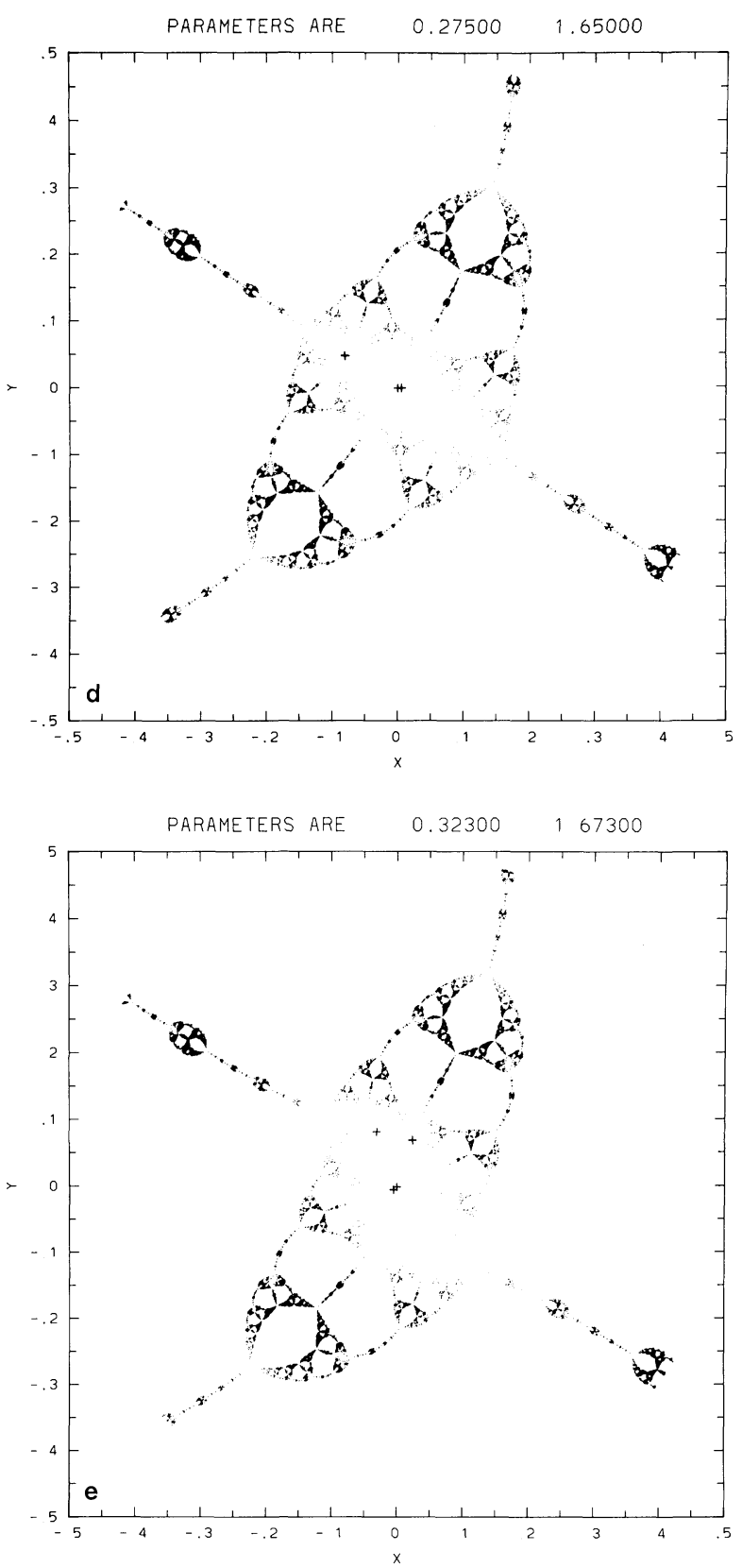

correspond(s) either to the location of the free critical point (or a few points on the stable periodic cycle if it is present). In all cases pictured, except Fig. 4.2f, one-half of the points on the periodic orbit are indicated; in the exceptional case the period is $440\left(2^{3} \cdot 5 \cdot 11\right)$ and no attempt has been made to accurately represent the cycle. 


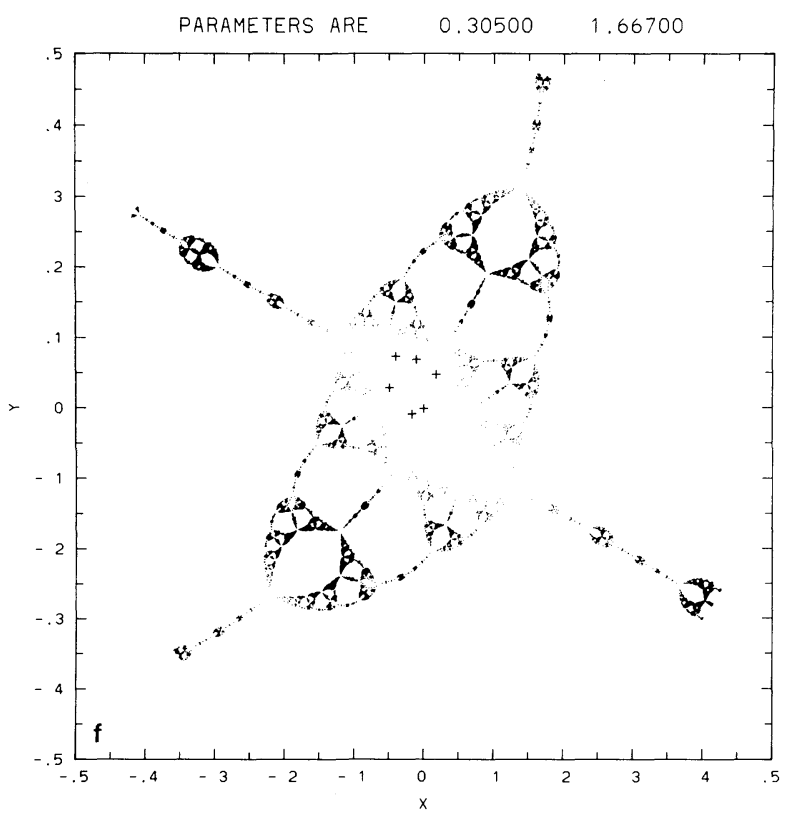

The most cursory comparison of Fig. 4.2e and f suggest that the structure of the Julia set has not undergone a radical change as parameters have varied even though the $A$-values are from markedly different regions of parameter space.

\section{Discussion}

In this article the study of Newton's transformation for the generic cubic polynomial was presented. For this iteration scheme two distinctly different types of behavior have been observed. In the first case Newton's method works for an open dense set of starting points. The exceptional set (Julia set) is closed, nowhere dense and has two dimensional Lebesgue measure zero. The second case exhibits an open set of initial conditions where Newton's method fails. The failure of the algorithm is due to the existence of a stable attracting period solution. The classical theory due to Fatou and Julia indicates when the iteration method should be expected to fail, i.e., when the orbit of a free critical point is attracted to a stable cycle.

Figure 3.4 indicates one of the parameter regimes where periodic behavior is obtained. A similar picture was first published by Mandelbrot [8] in the study of the iterations of (1.1). The reappearance of this figure in this study and in any general analytic family is explained by the behavior of the complex eigenvalue or derivative on the attracting periodic cycle.

In this study it was also noted that the Julia set of $N f_{A}$ shows an unexpected robustness, but that Julia sets for different islands are significantly different.

Acknowledgements. We would like to thank the National Center for Atmospheric Research, which is sponsored by the National Science Foundation, for providing computing time for this project. J. H. Curry was supported in part by NSF Grant PRM 8106833 and gratefully acknowledges that support. L. Garnett and D. Sullivan would like to thank the Mathematics Department of the University of Colorado, Boulder for its hospitality. 


\section{References}

1. Brolin, H.: Invariant sets under iteration of rational functions. Ark. Math. 6, 103-144 (1966)

2. Douady, A., Hubbard, J. : Itération des polynômes quadratiques complexes. C. R. Acad. Sci. Paris 294, 123-126 (1982)

3. Douady, A.: Systèmes dynamiques holomorphes. Séminaire Bourbaki 1982/83, No. 599

4. Fatou, P.: Sur les équations fonctionelles. Bull. Soc. Math. France 47, 161-271 (1919); 48, 33-94; 208-314 (1920)

5. Guckenheimer, J.: Endomorphism of the Riemann sphere. Proc. Symp. Pure Math. 14, 95-123 (1970)

6. Jakobson, M.V.: Structure of polynomial mappings on a singular set. Math. USSR Sb. 6, 97-114 (1968)

7. Julia, G.: Memoir sur l'iteration des function rationelles. J. Math. Pures Appl. 4, 47-245 (1918)

8. Mandelbrot, B.: Fractal aspects of $z \rightarrow \lambda z(1-z)$ for complex $\lambda$ and $z$. Ann. N.Y. Acad. Sci. 357, 249-259 (1980)

9. Mané, R., Sad, P., Sullivan, D.: On the dynamics of rational maps (preprint) (1982)

10. Sullivan, D.: Itération des fonctiòns analytiques complexes. C. R. Acad. Sci. Paris 294, 301-303 (1982)

Communicated by O. E. Lanford

Received January 20, 1983; in revised form June 1, 1983 
